\title{
Remote pandemic teaching in quantitative and instrumental chemical analysis courses at a Hispanic serving institution
}

\author{
Ángel L. Morales-Cruz ${ }^{1}$ (D) B Bonny M. Ortiz-Andrade ${ }^{2}$ (D) - Joselyn Del Pilar-Albaladejo ${ }^{1}$ (D) - Liz M. Díaz-Vázquez ${ }^{1}$ (i) $^{-}$ \\ Uriel Rivera-González ${ }^{1}$ (i) • Vilmalí López-Mejías ${ }^{1}$ (i)
}

Accepted: 18 February 2021 / Published online: 11 April 2021

(C) Springer-Verlag GmbH Germany, part of Springer Nature 2021

\section{Introduction}

The faculty and students of the University of Puerto Rico have endured many hardships over the past years; budget cuts, hurricanes, and earthquakes are among some. These prior scenarios have made both instructors and students resilient to instructional change. Thus, when the announcement was made regarding the transition to remote teaching, students and instructors braced for the challenge of adopting and adapting several teaching strategies to fit the new normal in higher education.

There are a multitude of well-studied teaching strategies easily adaptable to remote learning [1-7]. In choosing among these, it was important to recognize that however the transition was made, we needed to keep in mind the accessibility, cost, acquisition and implementation time frame, active learning potential, increased student engagement, and safety. This article focuses on our experiences as educators transitioning the Quantitative and Instrumental Chemical Analysis lectures and laboratories into remote learning as the COVID-19 pandemic evolved. We emphasize the challenges met in selecting resources, implementation hurdles confronted, and an evaluation of what we think were the most effective instructional practices to engage students in remote pandemic learning. The article discusses the course preparation and teaching strategies employed in the lecture and laboratory portion of these core courses.

This contribution is part of a series featuring teaching analytical science during the pandemic in order to support instructors in preparing their courses.

Vilmalí López-Mejías

vilmali.lopez@upr.edu

1 Department of Chemistry, University of Puerto Rico, Río Piedras Campus, San Juan, PR 00931, USA

2 Education and Outreach Program, Molecular Sciences Research Center, University of Puerto Rico, San Juan, PR 00926, USA

\section{Course demographics}

The Quantitative and Instrumental Chemical Analysis are semester courses typically taken consecutively by undergraduate students in their second or third year. Students enrolled (60\% female and 78\% Hispanic) in these courses are young $(75 \%$ are $<24$ years), and most $(80 \%)$ receive financial assistance [8]. The lecture component has an enrollment of 25-50 students per semester, while enrollment for the laboratory is limited to 24 students per session. The lecture component meets twice a week for $1 \mathrm{~h}$ and $20 \mathrm{~min}$, while laboratory meets twice a week for $3 \mathrm{~h}$.

\section{Course preparation}

To assess their remote learning resources, students were surveyed about their access to Internet services, hardware (laptops, desktop computers, or tablets), and software (scanning apps, PDF converter). They were also asked about their learning environment and time commitments. The questionnaire is provided as Supplementary Information. As a result of the questionnaire, students were pointed to on-campus resources that improved their accessibility to the Internet and devices. These actions minimized the effects of inadequate resources that some students might have confronted and offered them an opportunity to reflect on what they needed to learn remotely.

\section{Remote course lectures}

In the midst of a global pandemic, instructors turned to four evidenced-based teaching strategies; problem-based learning [5-7, 9], peer mentoring [10], case studies [3], and model-based learning [4] to deliver course content remotely. These active learning strategies were materialized using both synchronous and asynchronous tools for 
introduction, training, evaluation, and feedback as illustrated in Fig. 1. The following sections describe how each of these strategies was implemented and evaluated in the lecture component of the Quantitative and Instrumental Chemical Analysis courses.

\section{Synchronous course lectures}

The lectures were adapted in the sense that a video conferencing platform was now needed to enable a dialog among participants. The lecture occurred synchronously and was facilitated by a device that would allow the instructor to write and draw in real-time. For the Quantitative Chemical Analysis lecture, each discussion started with a "Challenge" that consisted of a complex problem that prompted the review of material covered in the previous lecture. Once solved individually or in a group of students, the next topic in the topic module was discussed. On the other hand, the Instrumental Chemical Analysis instructor intertwined a case study throughout the lecture as a strategy to apply concepts being developed directly to a social framework in which chemical problems might exist. Both of these components were shifted from in-person to remote pandemic teaching.

A key component in online lectures was student engagement. During lectures, the instructors would poll the students and the results were then used to guide the discussion and clarify doubts. Another way the instructors attempted to engage students was by incorporating their ideas making frequent use of polling and chat tools.

\section{Synchronous peer mentoring sessions and office hours}

During the Quantitative Chemical Analysis synchronous lecture, students could privately chat with each other or a peer mentor, who joined the discussion. Peer mentors are undergraduate students who have previously excelled in the course and receive one credit hour for tutoring and mentoring. It is recommended that the instructor remind students about the availability of the peer mentor to increase student engagement with this resource. Peer mentors were also in charge of reporting attendance and participation back to the instructor. In addition to the synchronous lectures, several $1-\mathrm{h}$ peer tutoring sessions occur synchronously throughout the week. Attendance for each session, although voluntary, was taken. These sessions help students address technical issues, clarify doubts, discuss past examinations, work through problem sets and serve as a place to socialize. The instructor also held office hours synchronously. Instructors note that the overall time for each topic module and office hours increased due to the use of technology to aid the discussion (i.e., idle time between screen sharing, entering/exiting the breakout rooms, solving audio/ connectivity issues). The ability for students to ask questions in real-time surpassed most of the discomfort related to Internet connectivity that often prevents instructors from using synchronous lectures.

\section{Asynchronous learning}

Asynchronous activities were included to reinforce learning, particularly for introductory concepts or application of

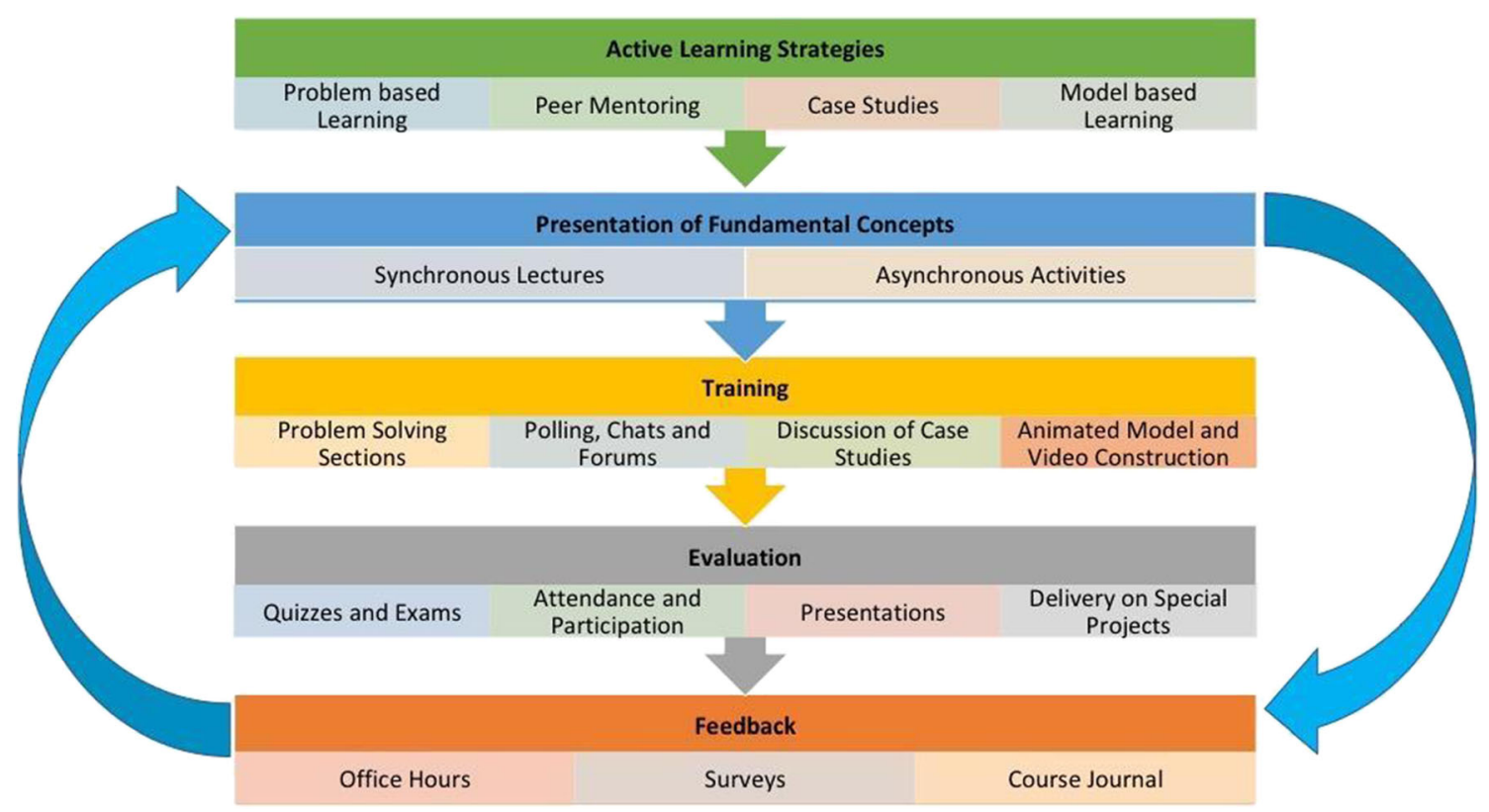

Fig. 1 Summary of the active learning strategies and how these were implemented and evaluated throughout the lecture component of the Quantitative and Instrumental Chemical Analysis courses 
instrumental techniques. The asynchronous component relied on pre-recorded videos explaining concepts, webinars, and podcasts about the application of instruments as well as supplementary readings and problems that complemented synchronous lectures. The instructor initiated a thread in the forum with open-ended questions, and the students followed by discussing doubts about the supplementary material assigned. Students who share devices, living spaces, and the Internet with other people or those who had unexpected responsibilities due to the pandemic, benefited from the asynchronous learning activities.

\section{Problem-solving sessions}

Remote problem-solving sessions were integrated every other week for a full lecture period or combined with the lecture material on the same day. At the start of the problem-solving session, the students were assigned randomly to breakout rooms to work cooperatively to complete the problem set. The instructor or a peer mentor intervenes when students use the "Ask for Help" button. Questions within the problem set were assigned randomly and each group was responsible for transcribing the solutions into a shared document that served as the answer key. The instructor or peer mentor would mark each particular solution as correct (green), partially correct (yellow), or incorrect (red) and comment on the answers as these became available. At the end of the lecture, the whole group met for $\sim 30 \mathrm{~min}$ and discussed the most challenging questions. If the time did not allow for a complete answer, the instructor and students discussed a strategy to solve the problem, and students were asked to come to office hours or attend the tutoring sessions.

To make this an effective instructional strategy, the instructor needs to provide students with clear and concise instructions (often at the top of the shared document), keep track of time, promote student participation, and lastly, allot time to discuss the outcome of the problem set. Remote problemsolving sessions take various iterations to get the right balance, both students and instructors need not be discouraged and recognize it is a learning process.

\section{Case studies}

Five case studies (Topics of the Case Studies are provided as Supporting Information) were employed as closing activities for the different course units in the Instrumental Chemical Analysis lecture. All cases have these common elements: a description of the problem, supporting information (tables/ graphs, scientific articles, or figures/videos), and a "decisionmaker" guided questions. These elements provide the students steps to develop solutions to the problem presented in the case study. Case studies are presented during the lecture using a video or newspaper article to promote the students' engagement. Then, students work in groups of 2-4 to answer the decision-maker-guided questions. To close the activity, each group presents their proposal, and the class comes to a consensus about the best strategy to solve the problem taking into consideration the following aspects of the instrumental analysis method; sampling, sample preparation, detection, analysis of results, advantages/disadvantages, and alternative approaches.

As demonstrated in previous studies [3], case studies helped our students to develop higher order analytical skills while discovering the usefulness of conceptual knowledge in solving real-world problems [11]. When contrasted to questions of similar difficulty presented during an in-person lecture to wrap-up course material, case studies increase the mean score on exam questions. An increase in student participation was noticeable during case studies. The instructor needed to be mindful in selecting cases that students could relate to increase their participation. Case studies should be adapted to fit this criterion.

\section{Construction of animated models and videos}

Modeling-based Learning (MbL) is a teaching and learning strategy whereby students engage in modeling while connecting with other practices such as asking questions, planning and carrying out investigations, analyzing and interpreting data, and constructing explanations to develop and refine explanatory accounts iteratively [4]. We included a special project using $\mathrm{MbL}$ to promote the understanding of instrumental techniques at the macroscopic and molecular levels. Simultaneously, the activity allowed expanding the number of techniques or applications discussed within the course. Students were required to work in pairs to develop a 5-10-min introductory video to explain an assigned instrumental technique. The video included a model or animation highlighting physicochemical principles of the technique and the function of the different parts of the instrument. Figure 2 shows some examples of the students' instrument models. As part of the assignment, students had to interact virtually with professors to understand how the instruments are used in diverse research areas. This interaction served two purposes: expose students to the real applications of the instrumental techniques and engage students in learning about the Department's research areas. The activity included three stages: (1) pre-production: the development of a script and identification of characters; (2) production: modeling, stage set-up, lighting, photography, and video generation; and (3) post-production: inserting narratives, sounds, and special effects. The students wrote a script and presented their initial model to the instructor during pre-production. At this stage, the instructor provided feedback and the opportunity to improve, if needed. Finally, at the end of the semester, the teams 


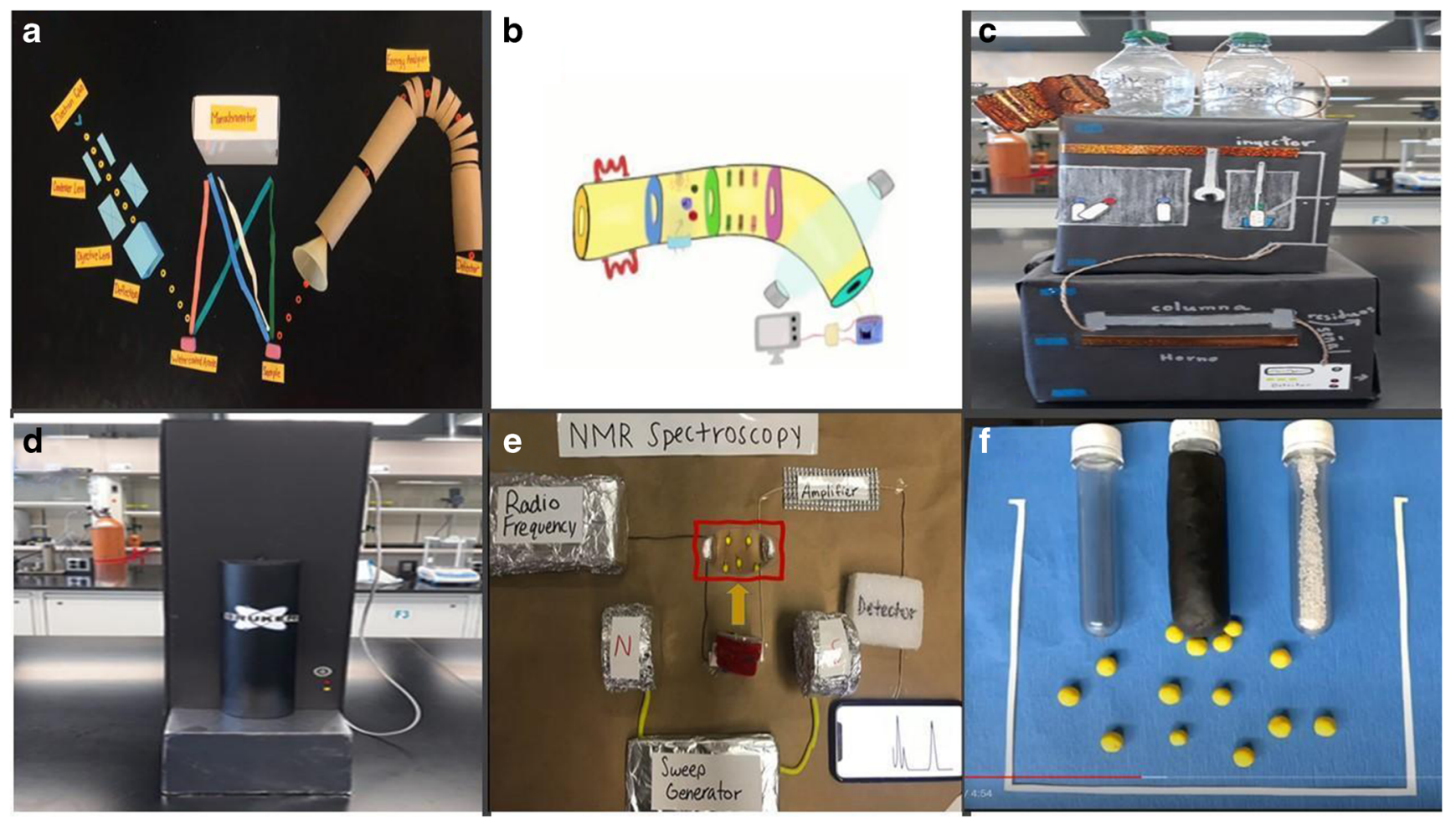

Fig. 2 Snap shots of animated instrument models and videos constructed by students. a X-ray spectrometer. $\mathbf{b}$ The analyzer in a mass spectrometer. c A high-performance liquid chromatograph. d A matrix-assisted laser

presented their creation to the entire group and answered questions during a synchronous lecture.

\section{Remote laboratory experiences}

In response to the global pandemic, instructors focused on those learning objectives that did not require performing inperson experiments. These are, students will (i) manage, process, and interpret experimental datasets using statistical analysis tools (ii) apply fundamental theories to explain experimental phenomenon, (iii) review and follow laboratory safety protocol, and (iv) evaluate laboratory procedures using the knowledge acquired in the lecture about the fundamental of chemical analysis. To this end, laboratory instructors implemented four evidenced-based teaching strategies; self-paced virtual experiments employing laboratory simulations, livestreamed and pre-recorded laboratory experiences, development of a research proposal, and model construction $[4,12]$. These strategies were enacted using both synchronous and asynchronous tools and are described in the following sections.

\section{Self-paced virtual experiments}

Self-paced virtual experiments (SPVEs) are guided experiential asynchronous practices performed in a virtual laboratory.
desorption/Ionization-time of flight mass spectrometer. e A nuclear magnetic resonance spectroscopy. f A potentiostat cell

This resource enables an experimental learning environment even in the absence of physical reagents or instruments [13] and it is available whenever and wherever with minimal intervention of teaching personnel. Different studies have suggested that SPVEs improve the understanding of difficult concepts and contribute to developing scientific inquiry selfefficacy $[12,14]$.

ChemCollective (www.chemcollective.com), a project of the National Science Digital Library developed to support chemistry education through interactive online activities, served as the virtual laboratory platform used for the Quantitative Chemical Analysis course. This platform is free, easy to implement, readily available, and safe. Three SPVE (Qualitative and Quantitative Analysis of Food Dyes, Designing a Buffer Solution with a Specific $\mathrm{pH}$, and Determining the Identity of an Unknown Solid Weak Acid) were incorporated. A summary of the instructional tools needed to realize SPVEs are shown in Fig. 3. The students work through the SPVE facilitated by an instruction sheet and a short discussion video that they could access through our instructional learning management platform, Moodle. The outcome is a worksheet that allows the students to record experimental details, gather data, and provide quantitative analysis and interpretation of the experimental results. For example, for the Qualitative and Quantitative Analysis of Food Dyes virtual laboratory, the students were tasked with determining the concentration of dyes present in a powdered 


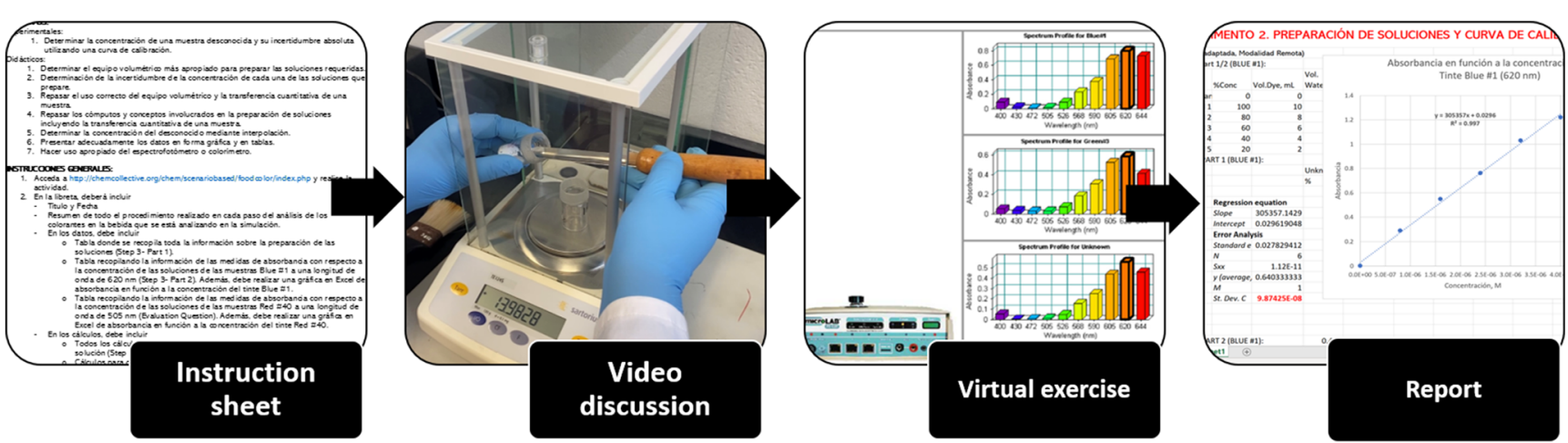

Fig. 3 A summary of the instructional tools needed to realize SPVEs: an instruction sheet, a short discussion video, the virtual exercise within ChemCollective, and a laboratory worksheet for data gathering, analysis, and interpretation

beverage. The activity included the separation of the dyes based on column chromatography, spectrum identification for each dye, preparation of standard solutions for the dyes, measurement of standard solutions to construct calibration curves, and measurement and determination of the concentration of dyes in the unknown sample with the appropriate statistical analysis.

Initial challenges were problems with comprehending the instructions provided and managing the platform's tools as students performed the first SPVE. To overcome these challenges, short discussion videos (not included at first) and more detailed instruction sheets accompanied later SPVEs. The videos helped students understand how to use the platform's tools and how to analyze the experimental data for all the subsequent SPVEs.

\section{Live-streamed and pre-recorded laboratory experiences}

Multimedia videos have been shown to be a highly effective educational tool for content delivery [15-17]. Three elements for video development [18] were considered to maximize their effectiveness: cognitive load, student engagement, and active learning. Teaching assistants and instructors for the Chemical Analysis laboratory worked on filming five laboratory video demonstrations (Calibration of Volumetric Equipment, Solution Preparation, Volumetric Analysis of the Concentration of $\mathrm{HCl}$, Potentiometric Analysis of a Phosphate Mixture, and Spectroscopic Determination of Mn in Steel). A typical video had a length of $\sim 15 \mathrm{~min}$ and showed the experimental procedure. It included views of the balance display and glassware scales so that the students could obtain the data directly from the equipment used.

For the Instrumental Chemical Analysis laboratory, video demonstrations for the following instruments were recorded and made available: spectrophotometers (i.e. UV-VIS, FTIR, AA), fluorometer, chromatography equipment (i.e. HPLCUV-VIs, GC-FID, GC-MS), sample preparation techniques (i.e. SPE, SPME), and differential scanning calorimetry. The videos included details that go from the instrument's calibration to the results' interpretation. The explanation of theory and step-by-step use of the analytical instrumentation revealed a significant improvement in the discussion component of the student's laboratory reports as well as grades compared with previous in-person laboratory experiences. This observation is consistent with previous literature in which the use of online videos is shown to increase the learning outcomes for difficult concepts [16].

Additionally, for this course the instructor live-streamed five laboratory experiences engaging students in the execution of experimental procedures to gather accurate data in real time. The experiences that were live-streamed were the Determination and Quantification of Ethanol in Vodka using FTIR, Synthesis and characterization of Silver Nanoparticles by UV-Visible Spectroscopy, Determination of Quinine of Tonic Water using Fluorescence, Quantification of Caffeine in Green Tea using UV-Visible Spectroscopy, and Determination of Water Hardness using Atomic Absorption Spectroscopy. A significant challenge the instructor faced in implementing the live-streaming was the visualization of the steps to use the instrument (i.e. focusing on the computer or instrument display, etc.) for a specific analysis. We believe that the laboratory video demonstrations, which are pre-recorded, helped to mitigate the pitfalls presented in the livestreamed experiments.

Although the overall use of pre-recorded and live-streamed laboratory experiences allowed us to keep providing students with experimental data and demonstrations of techniques, we faced a series of challenges that will inform future iterations of the course. Accountability was an issue, as students would skip the pre-recorded videos. We will work on the integration of questions within the videos and quizzes to assess viewer's comprehension and encourage the use of the pre-recorded videos for learning. In addition, we will develop shorter videos $(<5 \mathrm{~min})$ to ease comprehension and decrease the cognitive load [19]. The video demonstrations included upbeat instrumental music, which could serve as a distraction. We plan to substitute the music for a narration of the 
experiment to highlight critical steps of the analysis. Additionally, even if live-streaming and pre-recorded laboratory experiences seem to engage students in experiential learning during the pandemic, they fall short on developing the skills students need to appropriately manipulate laboratory equipment and instrumentation leading to the acquisition of high-quality quantitative data for analysis.

\section{Proposal writing}

Traditionally, students enrolled in the Quantitative and Instrumental Chemical Analysis laboratory develop a proposal, execute, and present their findings. To adapt this strategy into remote pandemic teaching, the students worked in teams developing a written proposal and, instead of its execution, they developed a hypothesis, presented the expected results, and proposed an analysis. A group of three students selected the topic for their project from a list provided by the instructor (Proposal Topics are Provided as Supplementary Information). For the Quantitative Chemical Analysis laboratory, the students were tasked with proposing a quantitative analysis using different techniques for a particular analyte in an array of real samples, such as determining the acidity of alcoholic beverages using potentiometric titration. For the Instrumental Chemical Analysis laboratory, students chose a particular problem to be solved using instrumentation that was previously covered as part of the laboratory experience. Laboratory instructors identified diverse themes as well as emergent problems for the topics of the proposals. For both courses, the proposals were written using the ACS guideline for Research Proposals for undergraduate students [20]. The objective was to have students apply what they learned, propose a method that included practical and technical considerations, propose a validation strategy, and describe in detail how they could prepare actual samples addressing removal interferences as a step in the sample preparation. The group submitted a draft for each component, and feedback was given throughout the semester based on predetermined deadlines. The instructor revised the documents, and each group presented the final proposal in a synchronous laboratory section.

The biggest challenge confronted by the students in working to develop their proposals during remote pandemic teaching was difficulties to identify (i) the analysis to be performed for a particular sample, (ii) expected results, and/or (iii) experimental errors. The casual interactions with the instructor, teaching assistants, and other students might have, during inperson teaching, solved these hurdles. In the face of remote pandemic teaching, the instructor had to schedule individual meetings with groups to discuss the projects. In the future, the instructor will provide an instruction sheet to the students to improve their ability to search the scientific literature. Overall, students did rise up to the challenge and some projects even integrated sampling of local sites.

\section{From users to builders: a DIY instrumental analysis laboratory}

This component of the laboratory included six-evidencebased teaching strategies: use of visuals in explanations, concept mapping, worked examples, practice, feedback, and group discussion. Students downloaded the assembly instructions and obtained the papercraft spectrophotometer from the Public Lab website (https://publiclab.org/wiki/foldable-spec). For the construction of the colorimeter, the main materials (cells, test tubes, volumetric flasks, dyes, pipettes, cardboard, and CDs) were picked up by students at a specific time and location. The pick-up resembled a drive-thru to minimize contact between instructors and students. Students were divided into groups to build two homemade spectrophotometers.

The construction of the first spectrophotometer was an adaptation of the work published by Public Lab's spectrometry project (https://publiclab.org/). Briefly, students use an old $\mathrm{CD}$, a box, and a fluorescent lamp to construct a spectrophotometer as the one shown in Fig. 4. Each member of the group had to fabricate their own equipment prior to the laboratory meeting in the breakout rooms; students came to a consensus about the best design. The design of this spectrophotometer allowed students to observe the components of the visible spectrum and get familiar with the concept of spectral calibration. Public Lab provided compact fluorescent lamps (CFLs) spectra as a reference to calibrate students' DIY spectrometers as shown in Fig. 4a, b. A set of questions was provided to the students to evaluate the construction of the DIY spectrophotometers and how they can improve spectral resolution (the questions are provided in the Supplementary Information). Most of the students were able to obtain $\sim 1$-nm resolution, after a few adjustments to their design (Fig. 4c).

For the second construction, students were asked to design a colorimeter employing household materials and their smartphone as the detector. First, they meet to brainstorm ideas and come with a proposal for the instrument design, including a justification for the selected materials. The instructor later met with each group and, through guided inquiry questions, pointed to the areas that need improvement. Figure 5 shows one of the colorimeters constructed by different groups. To validate the utility of the spectrophotometers, students used them to determine the concentration of food dyes in beverages employing BeerLambert Law. Standards of known concentration of the dyes (Allura red and Tartrazine) were provided for quality control and validation of the accuracy of the instrument and method. Although the instrument resolution and accuracy cannot be compared with that of commercial instruments, the DIY construction of spectrophotometers allows the students to understand the function of each component of this instrument. 

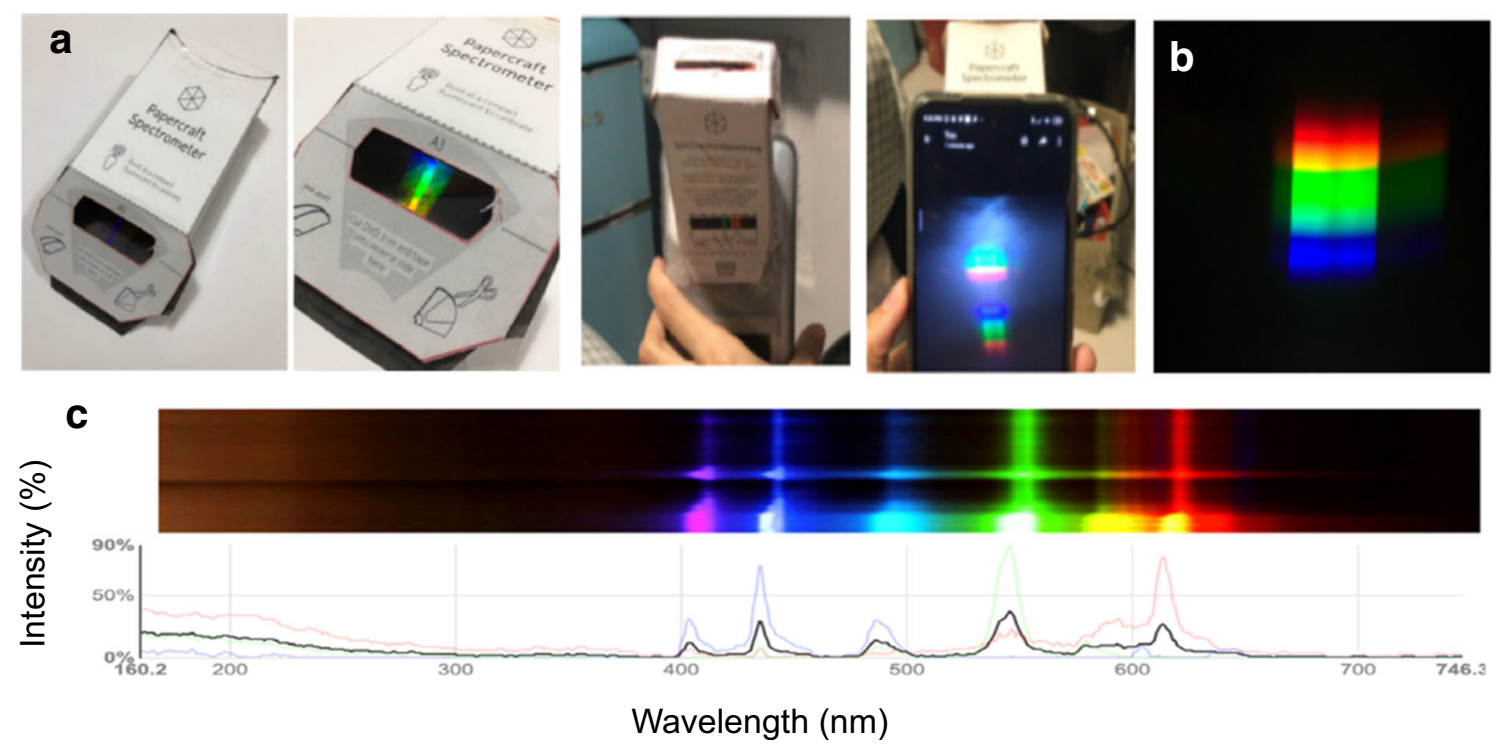

Fig. 4 Example of students' DIY spectrophotometer. a Construction following the Public Lab's spectrometry project template. b An image of the visible spectrum captured by a smartphone. c A calibration spectra for the spectrophotometer

\section{Overall outlook}

It is the authors' opinion that the Quantitative and Instrumental Chemical Analysis courses, particularly, their laboratory component, should not be offered remotely unless the risks outweigh the benefits for the students such in the case of the current global COVID-19 pandemic. In our opinion, there is no better alternative to acquire the analytical and technical skills needed to become professionally competent in the chemical sciences than to experience in-person Quantitative and Instrumental Chemical Analysis laboratories. The authors worry about the preparedness of students undertaking senior-

a

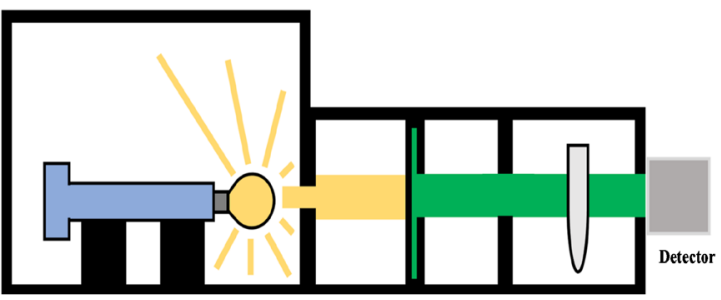

C

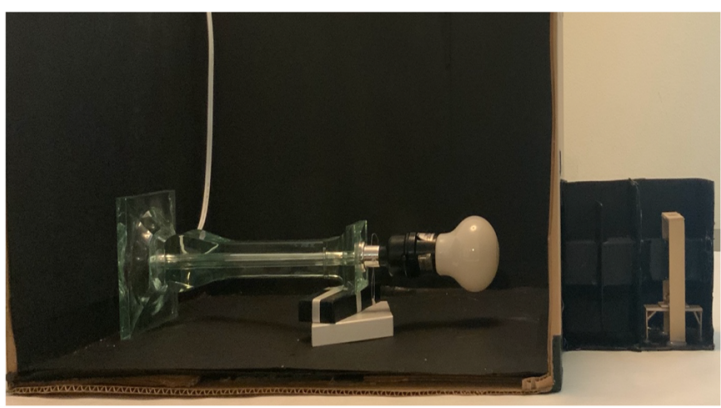

level courses, professional careers, or graduate school with minimal practical experience to complement their conceptual learning as the effects of the pandemic on remote teaching will linger at least through the summer months. Within the constraints of accessibility, cost, acquisition and implementation time frames, and safety, we resorted to implement evidencebased teaching strategies that would foster active learning such as case studies, problem-solving sections, MbLconstruction of models, SPVEs, laboratory demonstrations, and others presented herein. In addition, many experiments were performed with household materials, which facilitated the acquisition of some fundamental technical skills that are

b

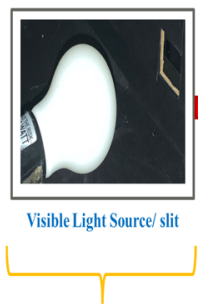

Box A
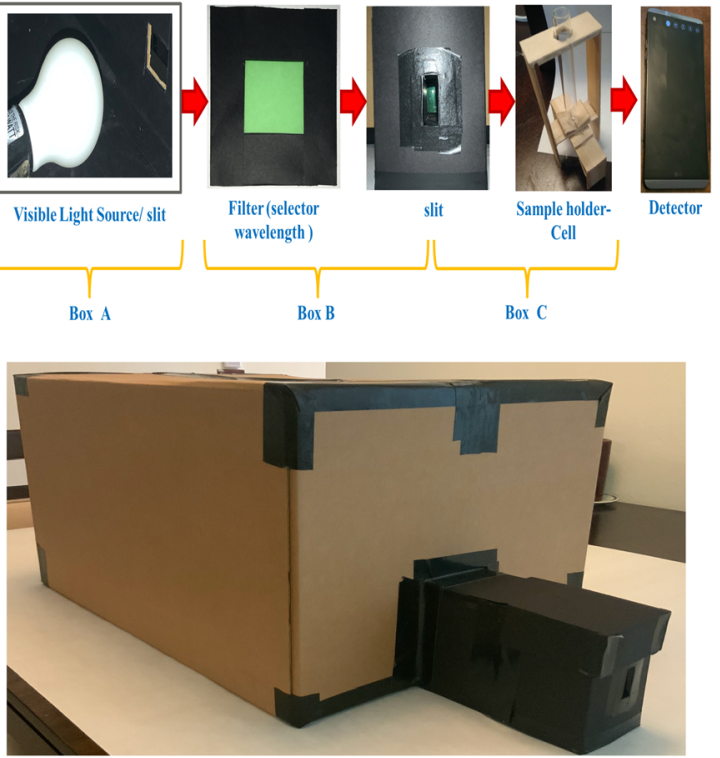

Fig. 5 Example of student-designed DIY colorimeter. a Diagram of the proposed colorimeter. b Selection of the materials for constructing the instrument. c Assembly of the instrument 
part of traditional laboratory experiences. These strategies buffered in a sense the impact of offering these experiential courses from afar. Moreover, these strategies were highly effective (in our opinion) in engaging the students in group work and social interactions that fomented motivation and appreciation for the chemical sciences as well as learning. Yet, the impact of the remote instruction for these core courses on the academic and professional preparedness of students should be investigated to mitigate any potential learning gaps.

From the instructor's perspective, the time needed for the development and implementation of remote courses surpassed that of traditional courses offered in person. One factor that contributed to the time spent in the planning and execution of remote teaching is the need to overcome technological barriers needed for the construction of highly engaging virtual learning environments that included, for example, storytelling as well as video recording and editing. Universities should support remote teaching by providing additional training, tools, and resources for educators to effectively engage students in remote learning.

Supplementary Information The online version contains supplementary material available at https://doi.org/10.1007/s00216-021-03243-5.

Authors' contribution Ángel L. Morales-Cruz (AMC), Joselyn Del PilarAlbaladejo (JDPA), Liz M. Díaz-Vázquez (LMDV), Uriel RiveraGonzález (URG), and Vilmalí López-Mejías (VLM) contributed to the conception and design of all remote learning experiences described. Bonny M. Ortiz-Andrade (BMOA) contributed to the conception and design of the animated models and videos developed for the Instrumental Analysis course. All authors contributed equally to the manuscript preparation. All authors read and approved the final manuscript.

Funding Partial financial support was received from the National Science Foundation's Center for Innovation, Research and Education in Environmental Nanotechnology (NSF-CREST, Grant No. HRD1736093). VLM and LMDV were participants in the Active Learning in Analytical Chemistry workshops funded by the National Science Foundation, through grant numbers DUE-1624898 and DUE-1624956.

\section{Declarations}

Conflict of interest The authors declare no competing interests.

\section{References}

1. Dekorver B, Chaney A, Herrington D. Strategies for teaching chemistry online: a content analysis of a chemistry instruction online learning community during the time of covid-19. J Chem Educ. 2020;97(9):2825-33.
2. Wang LQ, Ren J. Strategies, practice and lessons learned from remote teaching of the general chemistry laboratory course at brown university. J Chem Educ. 2020;97(9):3002-6.

3. Hibbard L. Case studies for general chemistry: teaching with a newsworthy story. J Chem Educ. 2019;96(11):2528-31.

4. Campbell T, Zhang D, Neilson D. Model based inquiry in the high school physics classroom: an exploratory study of implementation and outcomes. J Sci Educ Technol. 2011;20(3):258-69.

5. Larive CK. Problem-based learning in the analytical chemistry laboratory course. Anal Bioanal Chem. 2004;380(3 SPEC.ISS):357-9.

6. Cindy E. Hmelo-Silver1 2. Problem-based learning: what and how do students learn? Educ Psychol Rev. 2004;16(3):235-66.

7. Woodward AE, Weiner M, Gosser D. Highlights - projects supported by the NSF division of undergraduate education. J Chem Educ. 1993;70(8):651.

8. David ME, Amey MJ. National Center for Education Statistics. SAGE Encycl High Educ. 2020.

9. Gao S, Wang Y, Jiang B, Fu Y. Application of problem-based learning in instrumental analysis teaching at northeast Agricultural University. Anal Bioanal Chem. 2018;410(16): 3621-7.

10. Damkaci F, Braun TF, Gublo K. Peer Mentor program for the general chemistry laboratory designed to improve undergraduate STEM retention. J Chem Educ. 2017;94(12):1873-80.

11. Buckley P, Fahrenkrug E. The Flint, Michigan water crisis as a case study to introduce concepts of equity and power into an analytical chemistry curriculum. J Chem Educ. 2020;97(5):1327-35.

12. Kolil VK, Muthupalani S, Achuthan K. Virtual experimental platforms in chemistry laboratory education and its impact on experimental self-efficacy. Int J Educ Technol High Educ. 2020;17(1):1-22.

13. Jeschke S, Scheel H, Richter T, Thomsen C. On remote and virtual experiments in eLearning. J Softw. 2007;2(6):329-33.

14. Husnaini SJ, Chen S. Effects of guided inquiry virtual and physical laboratories on conceptual understanding, inquiry performance, scientific inquiry self-efficacy, and enjoyment. Phys Rev Phys Educ Res. 2019;15(1):10119. https://doi.org/10.1103/ PhysRevPhysEducRes.15.010119.

15. Stockwell BR, Stockwell MS, Cennamo M, Jiang E. Blended learning improves science education. Cell. 2015;162(5):933-6.

16. He Y, Swenson S, Lents N. Online video tutorials increase learning of difficult concepts in an undergraduate analytical chemistry course. J Chem Educ. 2012;89(9):1128-32.

17. Milligan JA. What is the value of synchronous engagement in small remote organic chemistry classes? Analysis of multiple-choice polling data from the COVID-impacted spring semester of 2020. J Chem Educ. 2020;97(9):3206-10.

18. Brame CJ. Effective educational videos: principles and guidelines for maximizing student learning from video content. CBE Life Sci Educ. 2016;15(4):es6.1-6.

19. Schacter DL, Szpunar KK. Stl-0000011.Pdf. Scholarsh Teach Learn Psychol. 2015;1(1):60-71.

20. Coghill AM, Garson LR, editors. ACS style guide: effective communication of scientific information. 3rd ed. Washington, DC.: American Chemical Society; 2006.

Publisher's note Springer Nature remains neutral with regard to jurisdictional claims in published maps and institutional affiliations. 


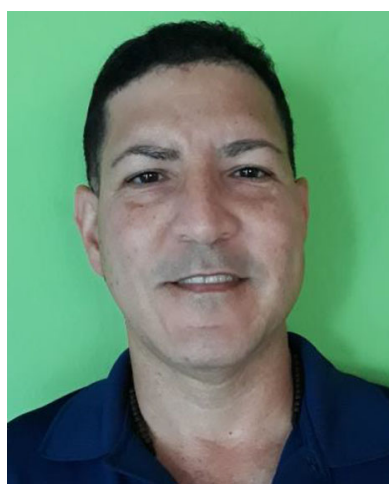

terials applied to aeronautics.
Ángel L. Morales-Cruz is an Assistant Professor in the Chemistry Department at the University of Puerto Rico, Río Piedras Campus. He served as instructor for several courses including General Chemistry, Physical Chemistry, Quantitative Analytical Chemistry, and Instrumental Analysis. He has also developed special topics courses on food and cosmetic chemistry at the undergraduate level. His research interests are focused on the development of ma-

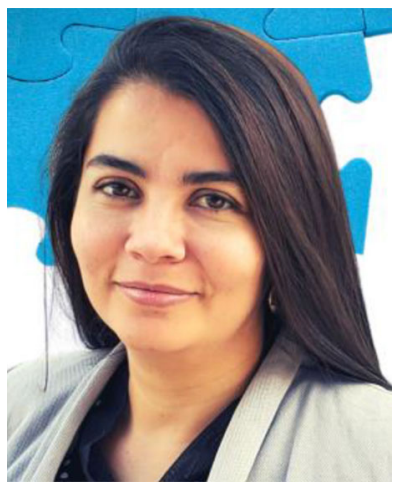

Bonny M. Ortiz-Andrade is an education and curricula development specialist focused on integrating science concepts into the community and the general public. Currently, she helps researchers develop activities and create educational materials as a Program Coordinator for Outreach and Education at the Molecular Sciences Research Center, and investigates best strategies to communicate scientific concepts effectively.

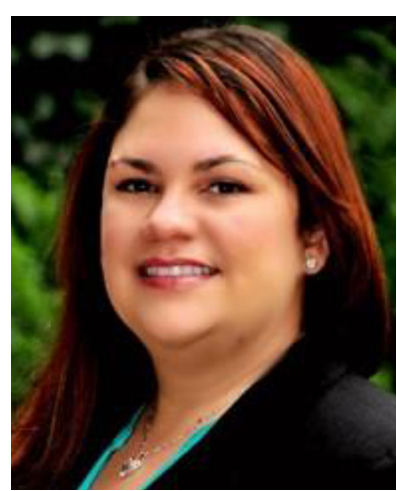

Joselyn Del Pilar-Albaladejo is an Assistant Professor in the Chemistry Departmentat the University of Puerto Rico, Río Piedras Campus. She teaches General Chemistry and Quantitative Analytical Chemistry courses for chemistry majors. Among her research interests is the development of functional inorganic materials for renewable energy, surface modification of nanocrystals, and characterization of fuel cells in operando conditions.

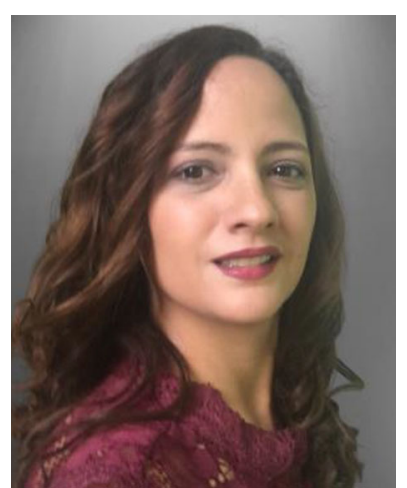

Liz M. Díaz-Vázquez is a full Professor and acting chair of the Chemistry Departmentat the University of Puerto Rico, Río Piedras Campus.She has taught different courses at the undergraduate level, including General Chemistry, Environmental Chemistry, Forensic Chemistry, Quantitative Analytical Chemistry, and Instrumental Analysis. Dr. Díaz-Vázquez research focuses on environmental metabolomics of marine ecosystems and the development of sustainable nanomaterials. She also directs several federally funded efforts to broaden participation and promote diversity in STEM disciplines through community engagement.

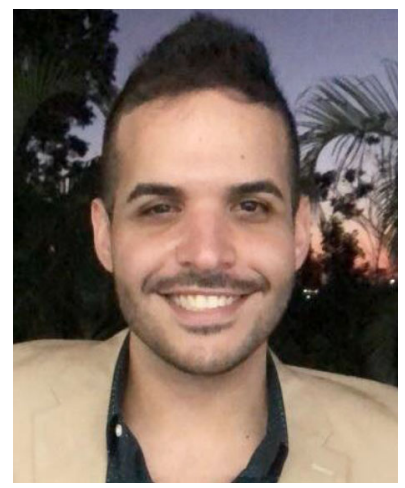

Uriel Rivera-González is an Assistant Professor in the Chemistry Department at the University of Puerto Rico, Río Piedras Campus. He teaches GeneralChemistry and Quantitative Analysis courses at the undergraduate level. Hisresearch interests focus on the development of electrochemical sensors, thestudy of the role of metal ions in neurodegenerative diseases, and the development and implementation of service-

Chemistry courses. based learning curricula in

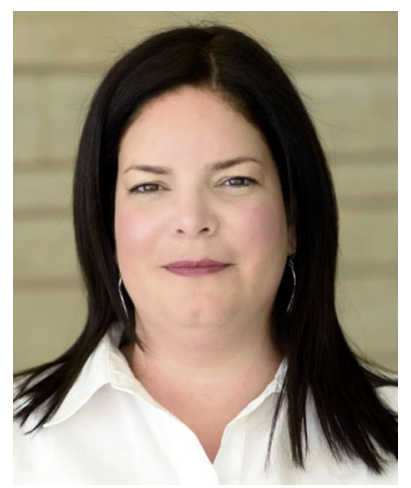

Vilmalí López-Mejías is currently an Assistant Professor in the Chemistry Department at the University of Puerto Rico, Río Piedras Campus. She has taught several courses at the undergraduate level ranging from General Chemistry to Quantitative and Instrumental Chemical Analysis. At the graduate level she teaches courses focused on Materials Science. Her research interests are focused on the application and mechanistic studies of polymer heteronucleants for the control and discovery of solid-state phenomena in organic compounds and their formulations. 